\title{
USING BPEL FOR BEHAVIOURAL CONCEPTS IN ODP ENTERPRISE LANGUAGE
}

\author{
Youssef Balouki, Mohamed Bouhdadi \\ A Department of Mathematics \& Computer Science, \\ University Mohammed V Morocco, MOROCCO \\ email:balouki@cmr.gov.ma \\ bouhdadi@fsr.ac.ma
}

\begin{abstract}
This paper studies the specification and the execution of behavioral concepts for Open Distributed Processing (ODP) Enterprise Language. The behavior of an ODP system is determined by the collection of all possible actions in which the system (acting as an object), or any of its constituent objects, might take part, together with a set of constraints on when these actions can occur. In order to specify the executable behavior of a system and to make the processes of the enterprise executable and controllable, the Reference Model for ODP $R M-O D P$ can be used as a meta-model for behavioral specifications. In the enterprise language the behavior is specified in terms of roles, processes, policies, and the relationships between these concepts. Firstly, we give the description and specification of the behavior by the activity diagrams. Secondly, we define the mapping from the concepts of behavior enterprise language to BPEL concepts and we present the syntax and the structure of a $B P E L$ Behavior process. Then we generate the corresponding BPEL and computational files to implement the specified process.
\end{abstract}

\section{INTRODUCTION}

The rapid growth of distributed processing has led to a need for coordinating framework for the standardization of Open Distributed Processing (ODP). The Reference Model for Open Distributed Processing (RM-ODP) [1-4] provides a framework within which support of distribution, networking and portability can be integrated. The foundations part [2] contains the definition of the concepts and analytical framework for normalized description of (arbitrary) distributed processing systems. These concepts are grouped in several categories. The architecture part [3] contains the specifications of the required characteristics that qualify distributed processing to be open. It defines a framework comprising five viewpoints, viewpoint language, ODP functions and ODP transparencies. The five viewpoints, called enterprise, information, computational, engineering and technology provide a basis for the specification of ODP systems.

Each viewpoint language defines concepts and rules for specifying ODP systems from the corresponding viewpoint. The ODP functions are required to support ODP systems.

The transparency prescriptions show how to use the ODP functions to achieve distribution transparency. The first three viewpoints do not take into account the 
distribution and heterogeneity inherent problems. This corresponds closely to the concepts of PIM (Platform Independent Model) and PSM (Platform Specific Model) models in the OMG MDA architecture.

However, RM-ODP can not be directly applicable [5]. In fact, RM-ODP only provides a framework for the definition of new ODP standards. which include standards for ODP functions [6-7]; standards for modeling and specifying ODP systems; standards for programming, implementing, and testing ODP systems.

We treated the need of formal notation for behavioral concepts in the enterprise language [8]. Indeed, the viewpoint languages are abstract in the sense that they define what concepts should be supported, not how these concepts should be represented. It is important to note that, RM-ODP uses the term language in its broadest sense: "a set of terms and rules for the construction of statements from the terms". It does not propose any notation to support the viewpoint languages. Using the Unified Modeling Language (UML)/OCL (Object Constraints Language) [9, 10] we defined a formal semantic for a fragment of ODP behavior concepts defined in the RM-ODP foundations part and in the enterprise language [11]. These concepts (time, action, behavior constraints and policies) are suitable for describing and constraining the behavior of ODP enterprise viewpoint specifications.

A part of UML meta-model itself has a precise semantic $[12,13]$ defined using denotational meta-modeling approach. A denotational approach [14] is realized by a definition of the form of an instance of every language element and a set of rules which determine which instances are denoted or not by a particular language element.

For testing ODP systems [2-3], the current testing techniques [15] [16] are not widely accepted. A new approach for testing, named agile programming [17] or test first approach [19], is being increasingly adopted. The principle is the integration of the system model and the testing model using UML meta-modeling approach [20] [21]. This approach is based on the executable UML [22].

In this context, OCL is used to specify the properties to be tested. The UML meta-models provide a precise core of any ODP tester.

In this context we use in this paper the BPEL (Business Process Execution Language for Web Services) (BPEL4WS or BPEL for short) to specify process behavior based on actions and policies in the context of ODP systems. The BPEL is an XML-based standard for defining how you can combine Web services to implement business processes. It builds upon the Web Services Definition Language (WSDL) and XML Schema Definition (XSD). This article specifies the behavior processes by the activity diagrams, and generates the corresponding BPEL and computational files to implement that process. This capability is used to highlight some benefits of the Object Management Groups (OMG) Model Driven Architecture (MDA) initiative: raising the level of abstraction at which development occurs; which, in turn, will deliver greater productivity, better quality, and insulation from underlying changes in technology.

The paper is organized as follows. Section 2 introduces, both BPEL and the core behavior concepts (time, action, behavior, role and process). Section 3 describes and specifies the behavior by the activity diagrams. In Section 4, we define the mapping from the concepts of behavior enterprise language to BPEL concepts and we present the syntax and the structure of a BPEL Behavior process. We focus on behavioral policies. A conclusion ends the paper. 


\section{PRELIMINARIES}

\subsection{BPEL}

BPEL, also known as BPEL4WS, build on IBM's WSFL (Web Services Flow Language) and Microsoft's XLANG (Web Services for Business Process Design). It combines the features of a block structured process language (XLANG) with those of a graph-based process language (WSFL). BPEL is intended to describe a business process in two different ways: executable and abstract processes. An abstract process is a business protocol specifying the message exchange behavior between different parties without revealing the internal behavior of any of them. An executable process specifies the execution order between a number of constituent activities, the partners involved, the message exchanged between these partners and the fault and exception handling mechanisms.

A composite service in BPEL is described in terms of a process. Each element in the process is called an activity. BPEL provides two kinds of activities: primitive activities and structured activities. Primitive activities perform simple operations such as receive (waiting for a message from an external partner), reply (reply a message to a partner), invoke (invoke a partner), assign (copying a value from one place to another), throw (generating a fault), terminate (stopping the entire process instance), wait (wait for a certain time) and empty (do nothing).

To enable the representation of complex structures, a structured activity is used to define the order on the primitive activities. It can be nested with other structured activities. The set of structured activities includes: sequence (collection of activities to be performed sequentially), flow (specifying one or more activities to be performed concurrently), while (while loop), switch (selects one control path from a set of choices), pick (blocking and waiting for a suitable message). The most important structured activity is a scope. A scope is a means of explicitly activities packaged together such that they can share common fault handling and compensation routines. It is composed of a set of optional fault handlers (exceptions can be handled during the execution of its enclosing scope), a single optional compensation handler (inverse some effects which happened during the execution of activities), and the primary activity of the scope which defines its behavior.

The sequence, flow, switch, pick and while constructs provide a means of expressing structured flow dependencies. In addition to these constructs, BPEL provides another construct known as control links which, together with the associated notions of join condition and transition condition, support the definition of precedence, synchronization and conditional dependencies on top of those captured by the structured activity constructs. A control link between activities $A$ and $B$ indicates that $B$ cannot start before $A$ has either completed or has been skipped. Moreover, $B$ can only be executed if its associated join condition evaluates to true, otherwise $B$ is skipped. An activity $X$ propagates a positive value along an outgoing link $L$ if and only if $X$ was executed (as opposed to being skipped) and the transition condition associated to $L$ evaluates to true. Transition conditions are Boolean expressions over the process variables. The process by which positive and negative values are propagated along control links, causing activities to be executed or skipped, is called dead path elimination. 
Figure 1 defines the BPEL core concepts [23]

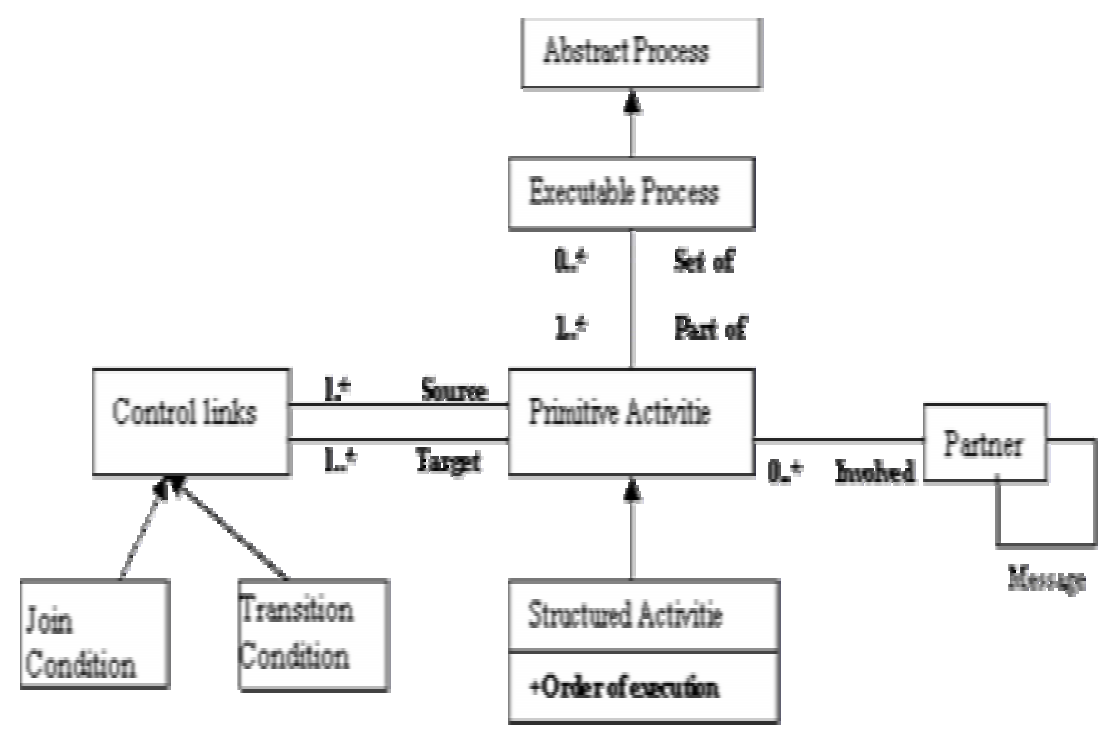

Figure 1 - Model of BPEL Core Concepts

\subsection{The Core behavioral Concepts in RM-ODP Foundations Part}

We consider the minimum set of modeling concepts necessary for behavior specification. There are a number of approaches to specify the behavior of distributed systems proposed by searchers with different background and considering different aspects of behavior. We use the formalism of the RM-ODP model, written in UML/OCL. We mainly use concepts taken from the clause 6 "Enterprise Language" of the RM-ODP. The behavior of a community is a collective behavior composed of the actions in which the objects of the community participate in fulfilling the roles of the community, together with a set of constraints on when these actions may occur, It may be interesting to specify which actor (enterprise object) initiates that action.

There are many specification styles for expressing when actions may occur (e.g. sequencing, pre-conditions, partial ordering, etc.). The actions and their ordering can be defined in terms of processes.

A process identifies an abstraction of the community behavior that includes only those actions that are related to achieving some particular sub-objective within the community. Each abstraction is labeled with a process name. The emphasis is on what the behavior achieves. Processes decompose the behavior of the community into steps. Its specification shall include specification of how it is initiated and how it terminates.

We represent a concurrent system as a triple consisting of a set of behavior, a set of process and a set of action. Each behavior is modeled as a finite or infinite sequence of interchangeable behavior and actions. To describe this sequence, there are mainly two approaches [24]. 
1. "Modeling systems by describing their set of actions and their behaviors".

2. "Modeling systems by describing their action spaces and their possible sequences of action changes".

These views are dual in the sense that a behavior can be understood to define action changes, and action occurring in action sequences can be understood as abstract representations of process. We consider both of these approaches as abstraction of the most general approach based on RM-ODP. We provide the formal definition of this approach that expresses the business process models.

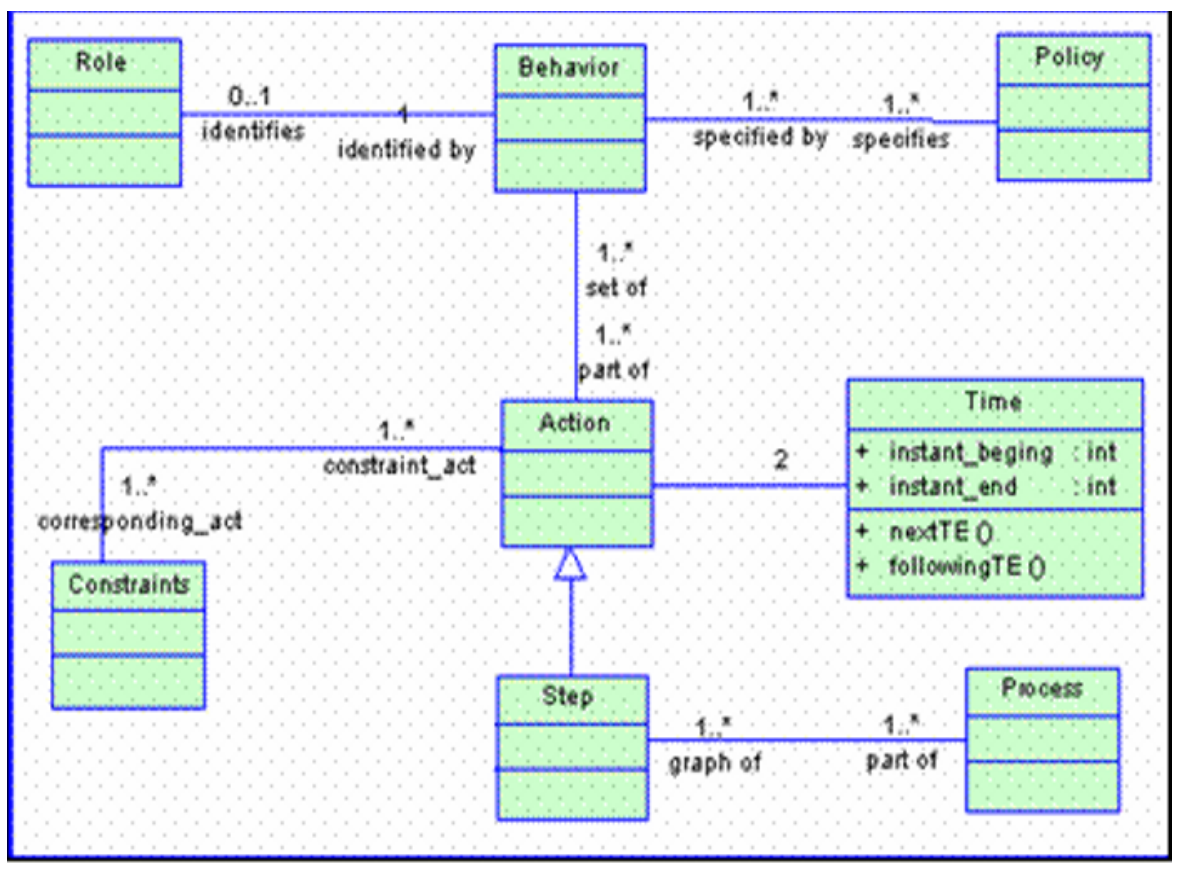

Figure 2 - Core Behavior Concepts

\section{UML PROFILE FOR AUTOMATED BEHAVIOR PROCESSES}

The ability to extend or customize UML is essential to MDA; UML can be customized to support the modeling of systems behavior. The scope of this article is mainly centered on stereotypes. Stereotypes are a way of categorizing elements of a model. We can combine a set of these stereotypes in a Profile. A UML Profile is used to define a specific set of extensions to the base UML in order to represent a particular domain of interest. For instance there are Profiles defined for CORBA and Data Modeling. A profile defines what elements of UML are to be used, how they may be extended, and any well-formedness rules to constrain the assembly of the elements. 
This section introduces a UML Profile which supports modeling with a set of semantic constructs that correspond to those in the Business Process Execution Language for behavior in enterprise language (see table 1).

Table 1 - Sample table

\begin{tabular}{|l|l|}
\hline Behavior Concepts & Profile Construct \\
\hline Process_El & $<<$ process $>>$ class \\
\hline Action & Activity graph on a $<<$ process $>>$ class \\
\hline Actor & $<<$ partner $>>$ class \\
\hline Policy & $<<$ process $>>$ class attributes \\
\hline Objective & Hierarchical structure and control flow \\
\hline$<<$ receive $>>,<<$ reply $>>$, & $<<$ receive $>>,<<$ reply $>>$, \\
$<<$ invoke $>>$ actions & $<<$ invoke $>>$ activities \\
\hline
\end{tabular}

We represent a subset of the UML profile through ODP trader [25] that defines a simple behavior process. It may be summarized as follows:

"ODP aims to provide distribution-transparent utilisation of services over heterogeneous environments. In order to use services, users need to be aware of potential service providers and to be capable of accessing them. Since sites and applications in distributed systems are likely to change frequently, it is advantageous to allow late binding between service users and providers. If this is to be supported, a component must be able to find appropriate service providers dynamically. The ODP trading function provides this dynamic selection of service providers at run time."

BPEL processes are stateful and have instances, so in BPEL this scenario is implemented as a behavior process which would have an instance for each actual behavior application being processed. Each instance has its own state which is captured in BPEL variables. In the UML profile, a process is represented as a class with the stereotype $<<$ Process $>>$. The attributes of the class correspond to the state of the process (variables in BPEL 1.1). The UML class representing the behaviorl process is shown in Figure 3.

\begin{tabular}{|c|}
\hline $\begin{array}{c}\Leftrightarrow \text { Ptocess } \Rightarrow \\
\text { BehawiotPtocess :BBhaviot }\end{array}$ \\
\hline $\begin{array}{ll}\text { + request } & \text { : actioninformation } \\
\text { tobjectiveinfo : be haviomo jective } \\
\text { +policy } & \text { : rulesbe havior } \\
\text { termor } & \text { : requestermor }\end{array}$ \\
\hline
\end{tabular}

Figure 3 - A UML class used to model a Behavior BPEL Process 
The behavior of the class is described using an activity graph. The activity graph for the behavior process is shown in figure 4 . The activities, such as invoke, are shown as the rectangles with rounded corners. The actions to be performed are shown as Entry conditions to the activity. For example, action constraint (a variable) is set to the result of the check service. The partners with which the process communicates are represented by the UML partitions (also known as swimlanes):Trader, Client and Server. The activities that involve a message send or receive operation to an partner appear in the corresponding partition. The arrows indicate the order in which the process performs the activities. Note that the assignment activity is not in a swimlane; it depicts an action that takes place within the process itself.

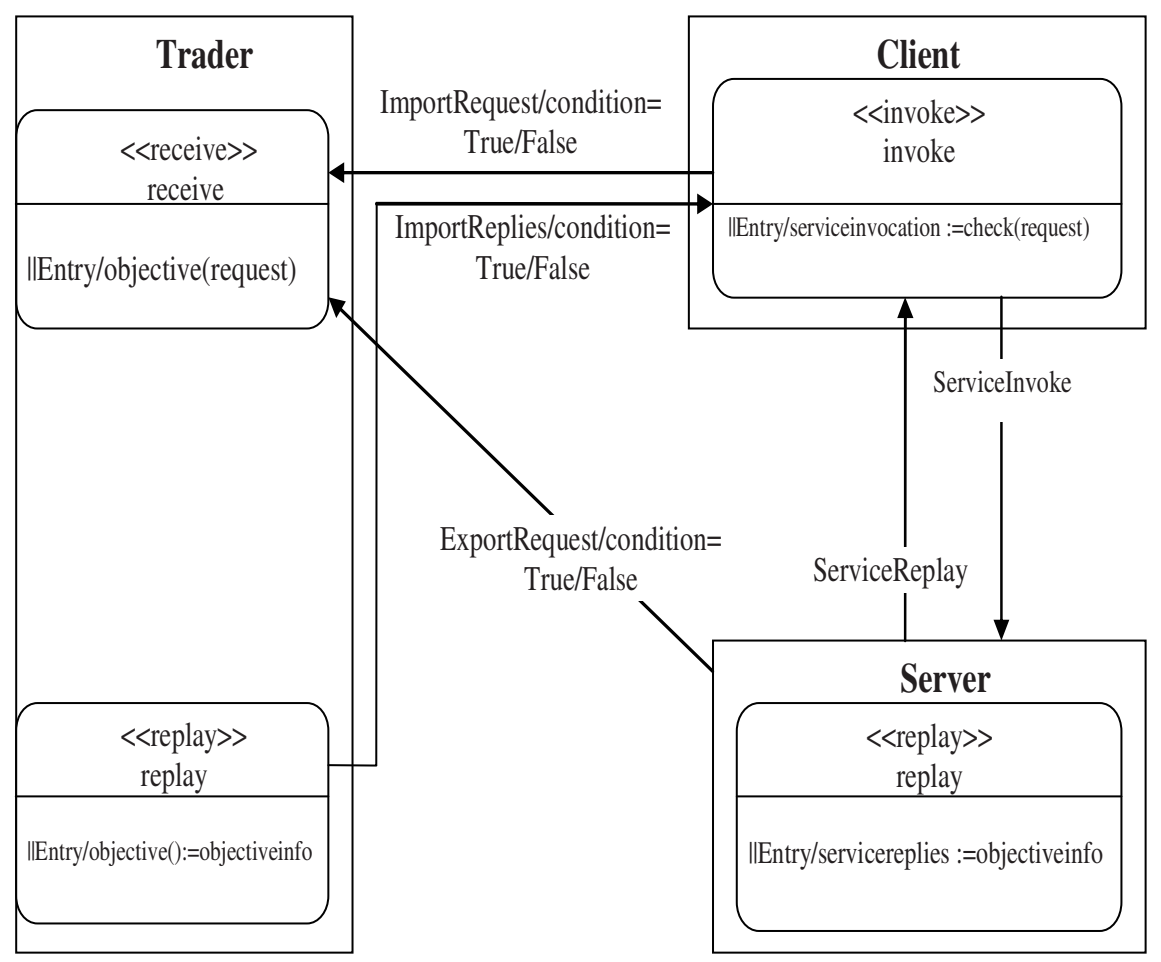

Figure 4 - An Activity Diagram for the Behavior Process

The reply activity returns a response back to the client, completing the execution of the process. Each activity has a descriptive name and an entry action detailing the work performed by the activity.

\section{MAPPING TO BPEL}

\subsection{From UML to BPEL}

The UML profile for automated behavior processes expresses that complete 
executable BPEL artifacts can be generated from UML models. Table 2 shows an overview of mapping from the profile to BPEL covering the subset of the profile introduced in this article.

Table 2 - UML to BPEL mapping overview

\begin{tabular}{|l|l|}
\hline Profile Construct & BPEL Concept \\
\hline$<<$ process $>>$ class & BPEL process definition \\
\hline Activity graph on a $<<$ process $>>$ class & BPEL activity hierarchy \\
\hline$<<$ process $>>$ class attributes & BPEL variables \\
\hline Hierarchical structure and control flow & $\begin{array}{l}\text { BPEL sequence and flow } \\
\text { activities }\end{array}$ \\
\hline$<<$ receive $>>,<<$ reply $>>,<<$ invoke $>>$ activities & BPEL activities \\
\hline
\end{tabular}

\subsection{Execution of the Behavior processes}

BPEL is an XML representation of an executable process which can be deployed on any process motor.

The atomic element of a process BPEL is an "activity", which can be the send of a message, the reception of a message, the call of an operation (sending of a message, makes an attempt of an answer), or a transformation of data.

A process BPEL defines, in XML, the activities realized by the framework of the behavior process execution. In the following we describe its structure and syntax.

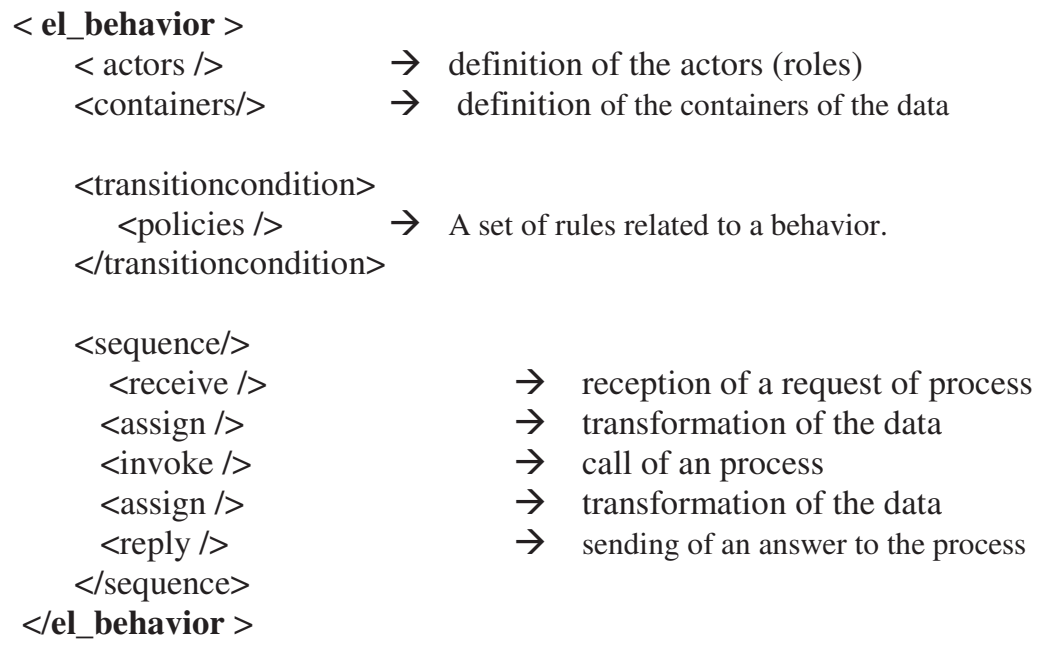

\section{$<$ process $>$}

$<$ partners />

$<$ containers $/>$

$\rightarrow$ definition of the partners (actions)

$<$ sequence / $>$

$<$ receive / $>$

$\rightarrow$ definition of the containers of the data

$\rightarrow$ reception of a request 


$\begin{array}{lll}<\text { assign } /> & \rightarrow & \text { transformation of the data } \\ <\text { invoke } /> & \rightarrow & \text { call of an action } \\ <\text { assign } /> & \rightarrow & \text { transformation of the data } \\ <\text { reply } /> & \rightarrow & \text { sending of an answer }\end{array}$

\section{$<$ /process $>$}

$<$ policies $>$ name $=$ "namepolicy"

$<$ process name $=$ "process" $/>$

$<$ actors name $=$ "actor" $/>$

$<$ choice $>$

$<$ policy type $=$ "obligations"/>

$<$ policy type $=$ "permissions"/>

$<$ policy type $=$ "prohibitions"/>

$<$ policy type $=$ "authorizations"/>

$</$ choice $>$

\section{$<$ policies $>$}

A cutdown version of the BPEL document that would be generated from the behavior process example is shown in Listing 1 (much of the detail is omitted here due to space constraints).

\section{Listing 1. Excerpt of the BPEL listing}

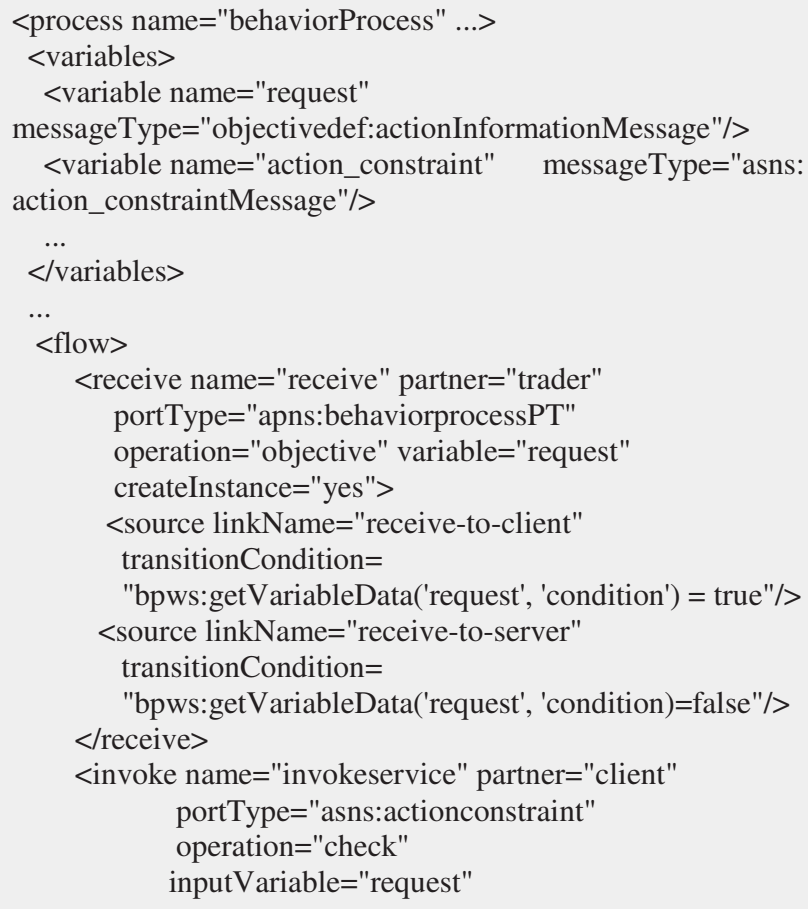




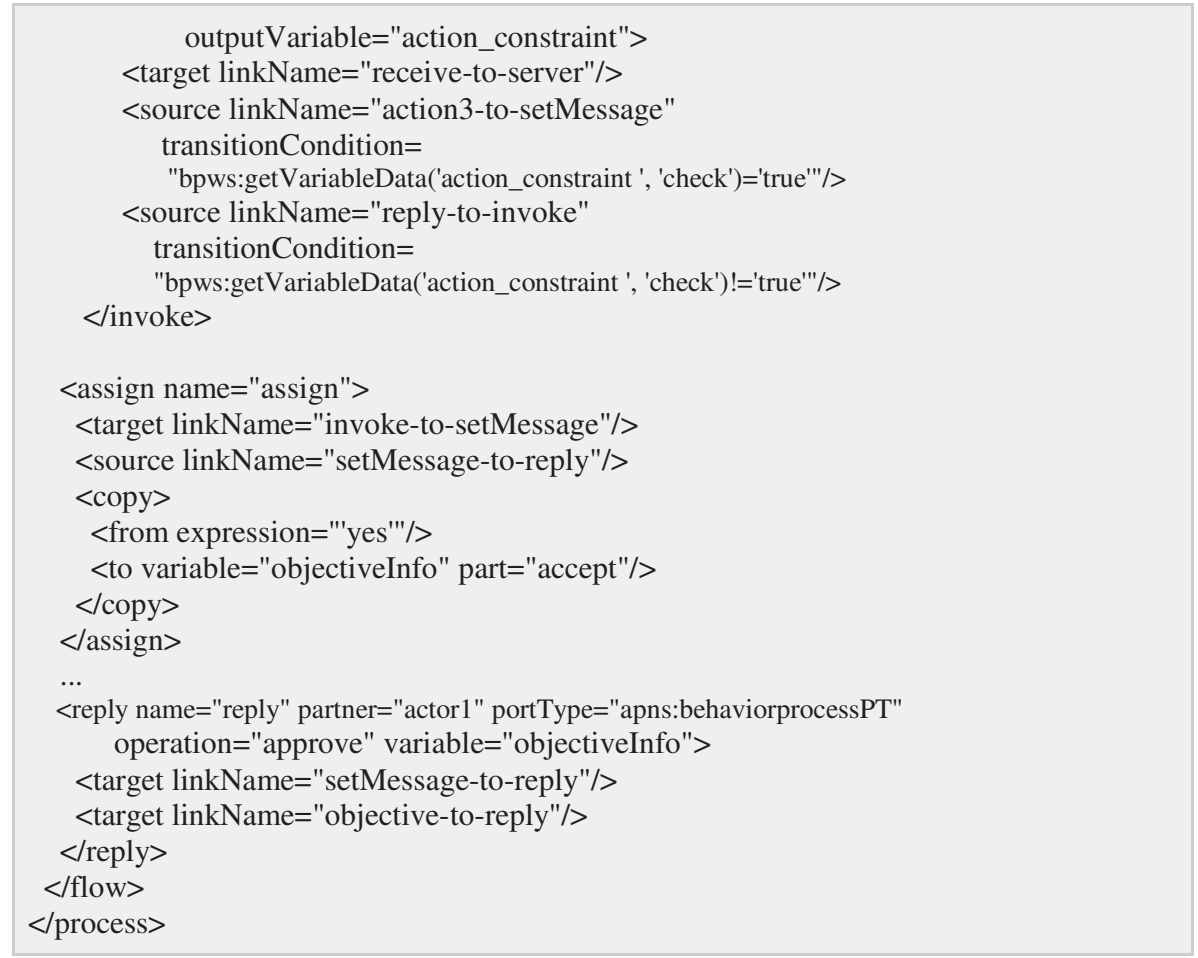

\subsection{The UML to BPEL Mapping Transformation}

The approach comes with a set of sample files for different scenarios [26]. The sample files are of two main types: UML model files which can be opened and modified with tools, and XML files containing the XMI version of the UML models and which are exported by theme. In figure 5, we can see that this corresponds to the UML models, or the XMI output of these tools.

Figure 5 uses a UML Activity Diagram to show the overall process of transforming the files; isn't UML useful? The boxes represent artifacts (usually files) while the ellipses represent an action or activity. The main stages are:

- Building and exporting the UML model to XMI (tools)

- Generating the BPEL, Actions, and behavior files

- Deploying these on the BPEL motor. 


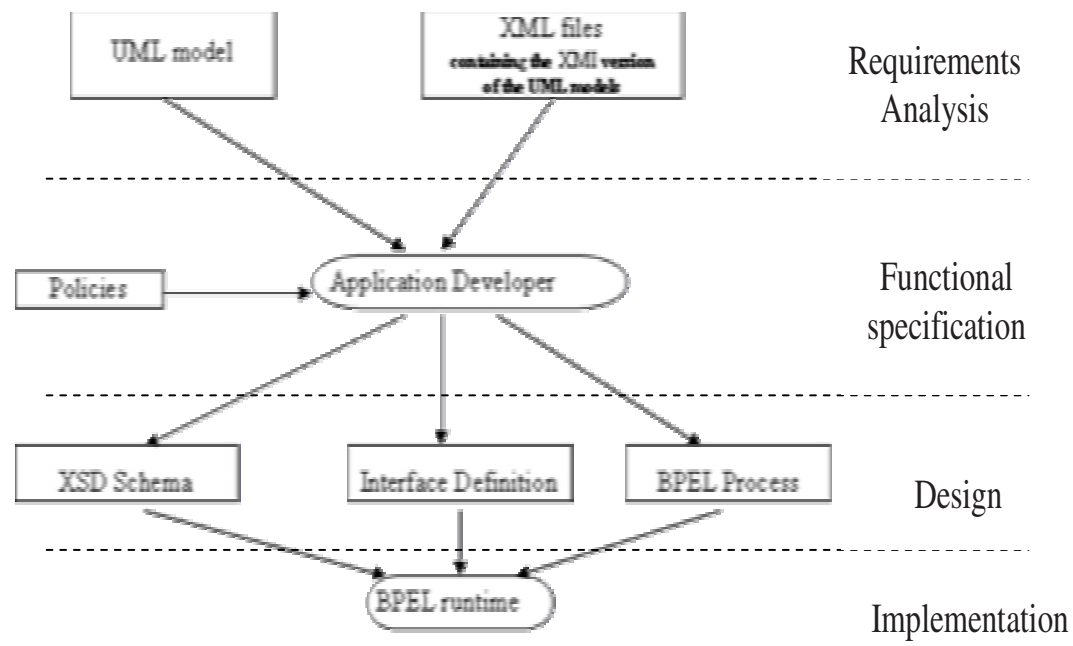

Figure 5 - Developing a process

\section{CONCLUSION}

This article has introduced a UML profile for automated behavior processes with a UML to BPEL translator. The profile allows developers to use normal UML skills and tools to develop behavior processes using BPEL. This approach enables serviceoriented BPEL components to be incorporated into an overall system design utilizing existing software engineering practices. Additionally, the mapping from UML to BPEL a model-driven development approach in which BPEL executable processes can be automatically generated from UML models.

Although we have only shown our method for the Trader behavior from the Enterprise Viewpoint, the method is generic enough to be applied in other viewpoints, such as trader from the Information Viewpoint.

\section{REFERENCES}

1. ISO/IEC, 'Basic RM-ODP-Part1: Overview and Guide to Use, 'ISO/IEC CD 10746-1, 1994

2. ISO/IEC, "RM-ODP-Part2: Descriptive Model, " ISO/IEC DIS 10746-2, 1994.

3. ISO/IEC, "RM-ODP-Part3: Prescriptive Model, " ISO/IEC DIS 10746-3, 1994.

4. ISO/IEC, 'RM-ODP-Part4: Architectural Semantics, ' ISO/IEC DIS 10746-4, July 1994.

5. M. Bouhdadi, et al. " An UML-based Meta-language for the QoS-aware Enterprise Specification of Open Distributed Systems, " Collaborative Business Ecosystems \& Virtual Enterprises, IFIP Series, Vol. 85, Springer Boston, pp.255-264, 2002.

6. ISO/IEC, "ODP Type Repository Function, " ISO/IEC JTC1/SC7 N2057, 1999.

7. ISO/IEC, The ODP Trading Function, ISO/IEC JTC1/SC21 1995.

8. ISO/IEC, 'RM-ODP Enterprise Langauge,’' ISO/IEC 15414, July 2006.

9. J. Rumbaugh, G. Booch, J. E. Jacobson, The Unified Modeling Language, Addison Wesley, 1999.

10. J. Warner and A. Kleppe, The Object Constraint Language: Precise Modeling with UML, Addison Wesley, 1998.

11. M. Bouhdadi, Y. Balouki, "Meta-modelling Semantics of Behavioral Concepts for Open Virtual Enterprises," ECC 2007, Athens 25-27 Sep, Springer Verlag (to appear 
12. S. Kent, S. Gaito, N. Ross, 'A meta-model semantics for structural constraints in UML, ', In H. Kilov, B. Rumpe, and I. Simmonds, editors, Behavioral specifications for businesses and systems, Kluwer Academic Publishers, Norwell, MA, September 1999. chapter 9.

13. E. Evans, R. France, K. lano, B. Rumpe, 'Meta-Modeling Semantics of UML, ' In H. Kilov, B. Rumpe, and I. Simmonds, editors, Behavioral specifications for businesses and systems, Kluwer Academic Publishers, Norwell, MA, September 1999. chapter 4

14. D.A. Schmidt, "Denotational semantics: A Methodology for Language Development, " Allyn and Bacon, Massachusetts, 1986.

15. G. Myers, 'The art of Software Testing, ', John Wiley \&Sons, 1979

16. R. Binder, " Testing Object Oriented Systems. Models. Patterns, and Tools, " Addison-Wesley, 1999

17. A. Cockburn, 'A Agile Software Development. "'Addison-Wesley, 2002.

18] B. Rumpe, "' Agile Modeling with UML, " LNCS vol. 2941, Springer, 2004, pp. 297-309.

19. K. Beck. Column on Test-First Approach. IEEE Software, vol. 18, no. 5, pp.87-89, 2001

20. L. Briand, 'A UML-based Approach to System testing, ' LNCS vol. 2185. Springer, 2001, pp. 194-208,

21. B. Rumpe, " Model-Based Testing of Object-Oriented Systems; "' LNCS vol.. 2852, Springer; 2003; pp. 380-402.

22. B. Rumpe, Executable Modeling UML. A Vision or a Nightmare?, In: Issues and Trends of Information technology management in Contemporary Associations, Seattle, Idea Group, London, pp. 697-701.

23. Dimitris Karagiannis et al. Business-oriented IT management developing e-business applications with E-BPMS,' ICEC 2007, 97-100

24. M. Broy, 'Formal treatment of concurrency and time,', Software Engineers's Reference Book, Oxford Butterworth-Henenmann (1991).

25. ISO/IEC. Information Technology - Open Distributed Processing - ODP Trading Function, ISO/IEC JTC1/SC21/N9122, August 1994.

26. keith_mantell," From UML to BPEL Model Driven Architecture in a Web services world” ,Report IT Architect, IBM 2003 